\title{
Violencia hacia mujeres con discapacidad en usuarias de un servicio de rehabilitacion en el año 2018
}

\section{Violence toward women with disabilities in users of a rehabilitation service in $\mathbf{2 0 1 8}$}

\author{
Rosa Ruffinelli ${ }^{a}$, Lilian Dominguez ${ }^{a}$, Celeste Houdin ${ }^{a}$
}

\begin{abstract}
Resumen
La investigación surge a partir de la necesidad de contar con información sobre este problema, invisibilizado en la sociedad. La condición de discapacidad en las que se encuentran las vuelve más vulnerables a sufrir violencia, la falta de estadísticas es un elemento que ayuda a desconocer las cifras reales de esta problemática social. Objetivo: Identificar las situaciones de violencia que viven mujeres con discapacidad en el ámbito doméstico, usuarias de un servicio de rehabilitación del sector público, a partir de un instrumento estandarizado de toma de datos. El principal resultado es que dé 119 mujeres con algún tipo de discapacidad el 53\% declara haber recibido maltrato de parte de las personas con quien viven, estos maltratos proceden en un $46 \%$ de familiares, el $40 \%$ manifiesta haber recibido en algún momento de su vida abuso o intento de abuso sexual, siendo el $23 \%$ de amigo o conocido y el $70 \%$ de otro familiar.

Palabras clave: discapacidad, violencia, abuso.
\end{abstract}

\begin{abstract}
The research arises from the need to have information about this problem, made invisible in society. The condition of the disability in which it is located in the social network of social problems. Objective: Identify situations of violence experienced by women with disabilities in the domestic sphere, users of a public sector rehabilitation service. The main result is that $119 \%$ declare to have been mistreated by the people with whom they live, these mistreatments come from $46 \%$ of relatives, and $40 \%$ have been received at some point of their abuse of life or attempt of sexual abuse, being $23 \%$ friend and acquaintance and $70 \%$ of another family member.
\end{abstract}

Keywords: disability, violence, abuse. a Universidad Nacional de Asunción, Facultad de Ciencias Sociales, Paraguay.

Correspondencia a: rruffinelli @yahoo.es

Recibido:

25 setiembre 2019

Aceptado:

17 diciembre 2019

Artículo publicado en acceso abierto bajo la Licencia Creative Commons.

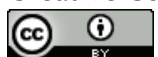

Cita:

Ruffinelli, R., Dominguez,

L., \& Houdin, C. (2019).

Violencia hacia mujeres con discapacidad en usuarias de un servicio de rehabilitacion en el año 2018. Kera Yvoty: social, 4, 41-48.

Trabajo realizado en función de docente investigador de la Facultad de Ciencias Sociales, UNA en el periodo 2018 , con fondos del Rectorado de la UNA. 
Ruffinelli, Dominguez, \& Houdin, C. Violencia hacia mujeres con discapacidad en usuarias de un servicio de rehabilitacion en el año 2018.

\section{Introducción}

Investigar temas referidos a la discapacidad nos sitúa por un lado ante personas con deficiencias en la mayoría de los casos con limitación física, mental o sensorial y por barreras que la misma sociedad les impone, que presentan en su mayoría altos grados de exclusión social, poco acceso a empleo, educación, accesibilidad a sitios públicos, entre otras desventajas sociales (Ruffinelli \& García 2018).

El tema de la discapacidad ha sido analizado desde diversas perspectivas en las ciencias sociales, con planteamientos que van desde una mirada deopresión impuesta, que se acentúa en una sociedad capitalista, Así se fue construyendo una matriz teórica que pudiera conceptualizar la discapacidad diferenciándola de la deficiencia. Cuando se habla de discapacidad se plantea que "es una forma de opresión impuesta por una organización social que, al no tener en cuenta las necesidades de las personas con "deficiencias", niega o restringe sus posibilidades de participación social, homologando sus condiciones de vida como grupo oprimido (Oliver \& Barnes, 2012, citando por Ferrante \& Duken 2016). Por otra parte desde el modelo anglosajón, se desarrollaron diversas mediaciones teóricas para entender la discapacidad, para las ciencias sociales significó "una bisagra en los modos de abordar la discapacidad" (Carolina Ferrante \& Juan Duken, 2017).

$\mathrm{Si}$ bien los años noventa dieron importantes avances en lo que hace a la discapacidad, si se asume una perspectiva marxista, la mirada de opresión y dominación estará referida principalmente en la condición de sujetos poseedores de los medios de producción y sujetos que solo tienen su fuerza de trabajo, para sobrevivir. Es decir esta sociedad en la vivimos establece diferencias concretas, donde condición de discapacidad viene a ser un elemento que se suma a la contracción capital/trabajo.

En el caso de la población en condición de discapacidad, la igualdad de oportunidades, la inclusión, la participación, aspectos estrechamente relacionados con la dignidad humana que se materializan en la libertad de las personas y en su proyecto de vida, son principios que no están garantizados efectivamente, por las instancias que deberían ser los principales propiciadores de ello, como lo son el estado, la sociedad y la familia. (Ruffinelli \& García 2018).

Cuando hablamos de violencia, no podemos hacer una abstracción de las bases estructurales de la sociedad en la que nos encontramos, por lo que se hace necesario comprender este problema social en la complejidad que adquiere, parafraseando a Iamamoto (2003) podemos decir que debemos entender la violencia, la pauperización y las formas de discriminación y exclusión social como una parte de la cara de una moneda, que lleva en la otra la concentración de la renta, de la propiedad y del poder. En este sentido amplio debe ser abordada la violencia. Una de los mecanismos que utiliza el sistema para lograr el control sobre las personas y de esta forma someterlas es el control social, control social que va desde el control del cuerpo, de la forma de pensar, de una estética, de una figura, entre otros. Otra forma menos visible de expresiones de dominación hace referencia a la violencia simbólica, trasmitida por ejemplo a través del lenguaje, de las costumbres, de cierto tipo de humor (chistes machistas, sobre pueblos originarios, sobre personas con discapacidad, entre otros)

La violencia contra la mujer funciona como un mecanismo para mantener la autoridad de los hombres. Cuando una mujer se ve sometida a la violencia, por ejemplo, por transgredir las normas sociales que rigen la sexualidad femenina y los roles de familia, la violencia no es sólo individual sino que, en virtud de 
sus funciones punitiva y de control, también refuerza las normas de género vigentes" (Alda Facio y Lorena Fries, 2005).

La violencia hacia las mujeres con discapacidad existe, como existe en el caso de las mujeres sin discapacidad. Desde esta premisa, se reivindica la importancia del tema a pesar de la total inexistencia de bases cuantitativas que puedan aportar alguna luz en el disperso y complejo mundo de la realidad en la que se desenvuelve esta problemática. Así, se considera que en la violencia hacia las mujeres tienen cabida los malos tratos físicos, junto con formas de amor, afecto, la reproducción, los abusos, el hostigamiento psicológico, la humillación, y que se da tanto en los casos de homosexualidad, heterosexualidad, etc.,

La violencia de género se considera un fenómeno psicosocial $y$, en ese sentido, influido tanto por el ambiente sociocultural como por las características de cada persona, que se expresa como una unidad: "basándose en la concepción de la persona como una unidad en la que se combinan estructuralmente lo biológico, lo psicológico, lo social y lo cultural" (Curcoll \& Vidal, 1992)

Convención Interamericana Para Prevenir, Sancionar y Erradicar la Violencia Contra la Mujer. "Convención de Belén Do Para" (1994) la define en el artículo 1 como "violencia contra la mujer a cualquier acción o conducta, basada en su género, que cause muerte, daño o sufrimiento físico, sexual o psicológico a la mujer, tanto en el ámbito público como en el privado, y en el artículo 2 "que violencia contra la mujer incluye la violencia física, sexual y psicológica: que tenga lugar dentro de la familia o unidad doméstica o en cualquier otra relación interpersonal, ya sea que el agresor comparta o haya compartido el mismo domicilio que la mujer, y que comprende, entre otros, violación, maltrato y abuso sexual; que tenga lugar en la comunidad y sea perpetrada por cualquier persona y que comprende, entre otros, violación, abuso sexual, tortura, trata de personas, prostitución forzada, secuestro y acoso sexual en el lugar de trabajo, así como en instituciones educativas, establecimientos de salud o cualquier otro lugar, y que sea perpetrada o tolerada por el Estado o sus agentes, donde quiera que ocurra.

La violencia basada en el género, se sostiene a través de la educación sexista que se imparte en la familia, la escuela, los medios de comunicación, la iglesia y otras instituciones sociales. En nuestro país las mujeres y las niñas siguen siendo las principales víctimas de la violencia. Esto es posible explicar debido al modo en que se establecen las jerarquías de poder en las relaciones sociales. La violencia es una estrategia de sometimiento presente y que se sigue trasmitiendo generacionalmente. Ésta se manifiesta de diferentes formas, en su expresión física, emocional, sexual y económica (Soto, González \& Elías, 2003)

Esta ausencia de expectativas sobre la proyección personal de una mujer con discapacidad y el hecho de no responder a las características que definen un rol social incrementa el desconcierto de los otros a la hora de relacionarse. Este hecho hace que, o bien renuncien a mantener esa relación, o lo hagan siempre bajo el condicionamiento de "no saber qué hacer" ante lo que creen es una persona diferente. Del mismo modo, esta actitud genera desconcierto en la mujer con discapacidad y lo que es peor acrecienta la inseguridad, el miedo y la valoración negativa de sí misma (Shum, Conde \& Iglesias, 1998)

Hablar de violencia contra las mujeres no es un problema menor, en el mundo entero miles de mujeres sufren a diario innumerables formas de violencia, las estadísticas que contamos no recogen datos diferenciados por violencia de género y discapacidad, lo cual es un indicador más de desigualdad. Las mujeres son el $50 \%$ de la población a nivel mundial, sin embargo, a pesar de la existencia de algunas normativas de protección sigue siendo la más vulnerada en todos sus derechos, y en algunos casos ni siquiera es considerada 
Ruffinelli, Dominguez, \& Houdin, C. Violencia hacia mujeres con discapacidad en usuarias de un servicio de rehabilitacion en el año 2018.

con derechos.

Según las declaraciones de la Phumzile Mlambo-Ngcuka, Secretaria General Adjunta de las Naciones Unidas y Directora Ejecutiva de ONU Mujeres, indica que para el año 2016 unas 60.0oo mujeres y niñas son asesinadas cada año, con frecuencia como una escalada de violencia doméstica.

En Paraguay SEDAMUR indica que en el año 2015 ha atendido un total de 1589 casos de violencia tanto, física, sicológica, sexual y económica, siendo la sexual 77 casos. Entre los años 2011 y 2015 se registran 285 casos de coaxión sexual a mujeres. En cuanto a los datos sobre homicidios de mujeres se tiene que en el 2016 el 55,6\% de las mujeres fueron, asesinadas por ser mujeres, y la mayoría de los asesinatos fueron cometidos por sus parejas o ex parejas sentimentales, el total de víctimas de ese año fue de 38 mujeres (Observatorio Nacional de Seguridad y Convivencia Ciudadana Ministerio del Interior 2016).

El InformeNacionaldeCumplimiento de la Convención Interamericana para la Eliminación de todas las Formas de Discriminación contra las Personas con Discapacidad (CIADDIS) y del Programa de Acción para el Decenio de las Américas por los Derechos y la Dignidad de las Personas con Discapacidad (PAD), ha recomendado al Paraguay (2016): fortalecer el sistema de recopilación de información que permita contar con datos y fuentes actuales y pertinentes sobre la situación de los derechos de las personas con discapacidad en el país, en ese sentido esta investigación es un primer relevamiento de la situación de violencia que sufren las mujeres con discapacidad que reciben servicios de rehabilitación.

\section{Materiales y Métodos}

Elobjetivoprincipaldelainvestigación fue: Identificar las situaciones de violencia que viven mujeres con discapacidad en el ámbito doméstico, usuarias de un servicio de rehabilitación.

\section{Objetivos específicos}

Describir los datos sociodemográficos de las mujeres con discapacidad, incluyendo su tipo de discapacidad. Identificar los tipos de violencia de la que son víctimas las mujeres con discapacidad en el ámbito doméstico. Identificar el o los principales agresores de las mujeres con discapacidad y los vínculos con la misma. Detectar el tipo de discapacidad con tipo de violencia más frecuente.

La investigación fue de tipo cuantitativo, exploratoria, a partir de un cuestionario estructurado y anónimo, con consentimiento informado. Las variables de estudio incluyeron datos sociodemográficos, tipos de discapacidad, nivel de dependencia (escala de Barttel) antecedentes de violencia física y sicológica con la escala de Gelles y Strauss. Para violencia psicológica o emocional, se utilizaron los ítems c, d, e f y g (desde el grito hasta las amenazas) y los ítems h, i, j, k, l, $\mathrm{m}, \mathrm{n}$ (desde empujar y zarandear hasta usar un arma para agredir) correspondientes a violencia física utilizado en la investigación titulada "Violencia y síntomas depresivos en estudiantes de Psicología" (Fresco, 2015) que contó con el permiso correspondiente de los autores para su utilización.

Para la realización de la investigación se contó con la anuencia de la autoridad institucional. Una vez concedido el permiso fueron socializados los instrumentos con los profesionales que colaborarían en la investigación.

El mismo fue aplicado por profesionales de Trabajo Social a todas las mujeres que acuden al servicio de rehabilitación, totalizando 119 unidades de análisis. Los criterios de inclusión considerados fueron que sean mujeres entre 18 y 60 años, que sean portadoras de algún tipo de discapacidad, ya sea física, sensorial o intelectual y que se encuentren en condiciones de responder al cuestionario y acepten participar del estudio. Las personas fueron informadas de los objetivos de la investigación y a partir del consentimiento informado, fue aplicado el 
cuestionario de forma confidencial sin la presencia de familiares, ni acompañantes, a fin de garantizar privacidad, el secreto y confidencialidad de la información. En el caso de personas con discapacidad auditiva se utilizó el lenguaje de señas. La Población fue tomada a partir de un corte de tiempo de dos meses, periodo en el que fueron socializados los objetivos de la investigación con los usuarios e invitados a participar. Un aspecto positivo en la difusión fue que las propias usuarias fueron las que promocionaron la investigación y realizaban la derivación de sus pares al departamento de trabajo social. Una vez finalizada la toma de datos se procedió a la carga en una planilla Excel, y posterior migración a SPSS, con el que fueron analizados los resultados.

\section{Resultados y Discusión}

La "violencia contra las mujeres es una forma de discriminación" señala el primer párrafo de la Recomendación General No. 19 del Comité CEDAW. La existencia de un vínculo directo entre violencia y discriminación está dada por "las relaciones de poder históricamente desiguales entre mujeres y hombres, que han conducido a la dominación de la mujer por el hombre" y se encuentra sustentada en el patriarcado (ONU Mujeres, 2016).

Si bien ninguna forma de violencia puede ser aceptada, una de las peores, por la doble discriminación que representa, que se encuentra oculta por las características propias de este colectivo es la violencia contra las mujeres con discapacidad. Y decimos que se encuentra oculta porque la discapacidad ha sido históricamente, para el estado, y para la sociedad un tema postergado.

La discapacidad es una dificultad que afecta a las sociedades, pues esta es el resultado de la incapacidad de las mismas de ser excluyentes y dar cabida a las diferencias individuales. Es decir, no son las personas con discapacidad las que deben de cambiar, sino las sociedades (Secretaría Nacional por los Derechos de las Personas con discapacidad, 2014).

Esta exclusión podemos identificar en la caracterización sociodemográfica de la población estudiada, en donde vemos que el $32 \%$ de las mujeres con discapacidad entre 18 y 60 años solo cursó hasta 6 años de educación formal (Tabla 1).

Los estudios sobre el ámbito de la violencia son limitados, y aún más en cuanto a la población de personas con discapacidad, debido a que la segregación especifica de datos sobre esta población en el país es todavía un desafío (Secretaría Nacional por los Derechos de las Personas con discapacidad, 2014).

En la muestra estudiada el $55 \%$ es portadora de discapacidad motora, el 16\% discapacidad auditiva, $17 \%$ discapacidad psicosocial, y 9\% (Tabla 1), lo que indica como algunas discapacidades por sus características son más visibles a otras y con ello también su vulnerabilidad a causa de su invisibilidad.

Un dato resaltante es que las mujeres con discapacidad auditiva en un $57 \%$ fue víctima de abuso o intento de abuso, y $47 \%$ víctima de maltratoy agresión. Con respecto a los distintos tipos de discapacidades, la intelectual es la más vulnerables a la hora de sufrir violencia, seguida de las mujeres sordas, aunque finalmente será el grado de afectación de la discapacidad y la autonomía que esta le permita tener a la mujer, lo que marque las principales diferencia (Gomiz, 2015).

Es interesante observar que hay mayor prevalencia de violencia psicológica que de violencia física sufrida y que los episodios de este tipo de violencia son más habituales, con relación a esto ya en el año 2013 el comité para la Convención por los Derechos de las Personas con Discapacidad en sus observaciones finales al estado paraguayo lamento que los delitos explotación, violencia y abuso hacia personas con discapacidad estén actualmente invisibilidades, debido a que las entidades operadoras de justicia no cuenten con registros sistematizados de los casos (Secretaría Nacional por los Derechos 
Ruffinelli, Dominguez, \& Houdin, C. Violencia hacia mujeres con discapacidad en usuarias de un servicio de rehabilitacion en el año 2018.

de las Personas con discapacidad, 2014).

En lo que respecta a los autores de la violencia, se puede identificar que, si nos centramos en la violencia psicológica las agresiones son por parte de alguien del entorno cercano, siendo las más habituales, los gritos y maltratos, como forma de relacionamiento hacia las mujeres con discapacidad y eses recurrente que, en las agresiones producidas dentro de la familia, la violencia provenga de los hijos cuando la madre tiene una discapacidad, que por la dependencia económica, la baja autoestima y la dependencia emocional, condicionan a la hora de romper situaciones de violencia. A esto se suma la dificultad en la accesibilidad tanto en las instituciones que deberían incorporar en sus servicios.

En lo que hace a los abusos o intento de abusos el $56 \%$ de están asociados a miembros de la familia, tíos, primos, padrastro, esto se encuentra muy vinculado a la condición de indefensión y dependencia en que se encuentran las mujeres con discapacidad, además de la carga de la cultura patriarcal que hace que toda mujer sea propiedad.

Otra de las características sociodemográficas de la población estudiada, es lo que hace a estado civil de las mujeres con discapacidad, el $57 \%$ de las mujeres con discapacidad es soltera o separada, esto explica (Shum, Conde \& Iglesias, 1998) como la incapacidad de la sociedad de relacionarse con lo diferente, y a muchas veces ante la ausencia de expectativas sobre la proyección personal de una mujer con discapacidad y el hecho de no responder a las características que definen un rol social incrementa el desconcierto de los otros a la hora de relacionarse.

Si bien a nivel nacional el acceso de las personas con discapacidad es aún bajo, según los datos recabados el $47 \%$ de la población estudiada tiene ingreso propio y de estos el $62 \%$ lo tiene por trabajo, mientras que las restantes tienen por subsidio u otros. Es importante resaltar este aspecto, aunque no hay diferencia de mayor o menor maltrato o agresión entre mujeres que tienen ingresos o no, el ingreso representa una forma de igualdad material para la toma de decisiones, y para el desarrollo de una vida plena.

Tabla 1

Grupo de edad y nivel educativo de las mujeres

\begin{tabular}{llr}
\hline & 19 a 29 años & \\
& 30 a 39 años & $17,6 \%$ \\
Grupos de Edad & 40 a 49 años & $2,7 \%$ \\
& 50 a 59 años & $21,8 \%$ \\
& Más de 6o años & $27,7 \%$ \\
\cline { 2 - 3 } Total & $10,1 \%$ \\
\hline \multirow{5}{*}{ Nivel de } & Ninguno & $100,0 \%$ \\
Educación & Primaria Incompleta & $1,7 \%$ \\
& Primaria Completa & $13,4 \%$ \\
& Secundaria Incompleta & $18,5 \%$ \\
& Secundaria Completa & $20,2 \%$ \\
& Edu. Media Incompleta & $12,6 \%$ \\
& Edu. Media Completa & $12,6 \%$ \\
& Universitaria Incompleta & $5,0 \%$ \\
& Universitaria Completa & $6,7 \%$ \\
\cline { 2 - 3 } & Total & $9,2 \%$ \\
\hline
\end{tabular}

Los resultados más relevantes de la investigación.

- El 32\% de las mujeres con discapacidad entre 18 y 60 años solo curso hasta 6 años de educación formal.

- El $57 \%$ de las mujeres con discapacidad es soltera o separada.

- $\quad$ El 88\% tiene independencia relativa, según la escala de Barthel,

- El 55\% de la población estudiada es portadora de discapacidad motora

- $\quad$ El $47 \%$ tiene ingreso propio.

- El 62\% de las mujeres con discapacidad que tienen ingreso propio lo tienen por trabajo y solo el $14 \%$ por subsidio.

- $\quad$ El $72 \%$ en algún momento le tiraron o rompieron algún objeto valioso

- El 95\% recibió maltrato o agresiones de personas con las que convive o le cuidan.

- El 32\% recibió amenaza de ser golpeada o recibir algún castigo

- El 12 \% declara haber recibido 
zarandeo o empujón.

- El 40\% alguna vez en su vida recibió intento o abuso de parte de hombre o mujer.

- Los abusos o intentos de abuso del 39\% fueron antes de los diez años

- Los abusos e intento de abuso del $48 \%$ fueron entre los once y 15 años

- El 23\% de los abusos o intento de abuso se dio por amigos o conocidos de la familia.

- $\quad$ El $56 \%$ de los abusos o intento de abusos están asociados a miembros de la familia, tíos, primos, padrastro.

- $75 \%$ de las mujeres con discapacidad en los últimos 5 años ha tenido pareja, novio o marido.

- $\quad$ El 50\% de las mujeres que en los en los últimos 5 años ha tenido pareja, novio o marido recibió gritos de parte de estos.

- El $21 \%$ de las mujeres con discapacidad que en los últimos 5 años ha tenido pareja, novio o marido, recibió amenaza de ser golpeada o recibir algún castigo.

- El 13\% recibió agresión física ( empujon o zarandeo) de parte de su pareja, novio o marido.

- El $52 \%$ las mujeres con discapacidad entre 19 y 29 años fueron víctimas de maltrato y agresión, mientras que las mujeres con discapacidad de 30 a 39 años el $63 \%$.

- $\quad$ El 53\% de las mujeres que tienen ingresos propios fueron víctimas de maltrato o agresión y el $54 \%$ de las que no tienen ingreso también, por lo que no hay relación entre las mujeres con discapacidad que tienen ingresos y las que no tienen.

- El 57\% de las mujeres con discapacidad auditiva sufrió abuso o intento de abuso.
- El $47 \%$ de las mujeres con discapacidad psicosocial sufrió abuso o intento de abuso.

- El $57 \%$ de mujeres con discapacidad entre 19 y 29 años sufrió abuso o intento de abuso.

- El 16\% de las mujeres con discapacidad, reciben maltrato psicológico (gritar, romper o patear objetos frecuentemente y muy frecuentemente) de los cuidadores $\mathrm{o}$ personas con quienes convive,

- La forma de maltrato más frecuente en las relaciones de pareja es la psicológica, $11 \%$ en forma frecuente y muy frecuente recibe gritos.

\section{Conclusiones}

Pese a que el Comité recomienda al Estado paraguayo implemente de manera urgente medidas efectivas para la identificación, la prevención y la protección necesarias para combatir las múltiples formas de discriminación y violencia que enfrentan las mujeres y niñas con discapacidad, en particular mujeres y niñas con discapacidad intelectual y psicosocial , y auditiva (Naciones Unidas, 2013) en el año 2018 aún no se cuenta con información estadística que dé cuenta de la situación de violencia hacia mujeres con discapacidad

Esta primera aproximación de violencia hacia mujeres con discapacidad refleja en su condición socioeconómica, el nivel de baja escolaridad, el estado civil predominante que es soltera o separada, y que casi la mitad no cuenta con ingreso propio, por lo que su situación es de dependencia económica. Las que acuden al servicio de rehabilitación son portadoras de discapacidad motora, más de la mitad.

El tipos de violencia de la que son víctimas las mujeres con discapacidad en el ámbito doméstico es la violencia psicológica, caso el total de la población estudiada manifiesta recibir este tipo de maltrato verbal, en forma frecuente o muy frecuente, por miembros de su familia. 
Los agresores son parte del núcleo familiar, esta misma conducta se repite en las relaciones de pareja, en donde más de la mitad es víctima de abuso psicológico.

En cuanto a la relación entre tipo de violencia y tipo de discapacidad se observó que la discapacidad psicosocial es víctima en mayor número de violencia psicológica en tanto las mujeres con discapacidad auditiva de abuso o intento de abuso.

\section{Agradecimientos}

A las mujeres con discapacidad que abrieron sus heridas para aportar a este estudio y visibilizar la violencia de que son victimas.

\section{Referencias Bibliográficas}

Agencia de los Derechos Fundamentales de la Unión Europea (2014). Violencia de género contra las mujeres: una encuesta a escala de la UE.

CERMI. (2010). Contribución del CERMI al estudio analítico sobre la violencia contra las mujeres y las niñas y la discapacidad (A/HRC/RES/17/10).
Convención de Belén Do Para. (1994). Convención Interamericana Para Prevenir, Sancionar y Erradicar la Violencia Contra la Mujer.

Curcoll, M. L., \& Vidal, J. (1990). Sexualidad y relación de pareja. Madrid: Minusval.

Gomiz, M. D. (2015). Violencia contra las mujeres con discapacidad (Tesis doctoral). UNED.

Mara Danel, P. (2018). Trabajo Social, Discapacidad. Intervenciones, Trayectorias y temporalidades. Entre Rios: Fundacion la hendija.

Naciones Unidas. (1995). Cuarta Conferencia Mundial de la Mujer, Declaración y Plataforma Acción de Beijing.

Naciones Unidas. (2013). Observaciones finales sobre el informe inicial del Paraguay, aprobadas por el Comité en su noveno periodo de sesiones. Ginebra. http://docstore.ohchr.org/ SelfServices/

\section{Sobre las Autoras \\ Rosa Ruffinelli}

Trabajadora Social, por la Universidad Nacional de Asunción, Máster en Trabajo Social y Doctorando en Educación por la Universidad Nacional de Asunción. Tutor de tesis de la FENOB/UNA. Docente de la Materia Abordaje Profesional y docente investigador FACSO, UNA.

\section{Lilian Dominguez}

Doctorando en Educación por la Universidad Nacional de Asunción, Máster en Trabajo Social. Coordinadora de Tesis de la Facultad de Ciencias Sociales de la Universidad Nacional de Asunción.

\section{Celeste Houdin}

Trabajadora Social por la Universidad Nacional de Asunción. Investigadora y feminista. Docente de la Facultad de Ciencias Sociales de la Universidad Nacional de Asunción. 